\title{
Genetic evidence for causal relationships between maternal obesity-related traits and birth weight
}

\author{
A full list of authors and affiliations appears at the end of the article.
}

\section{Structured abstract}

Importance-Neonates born to overweight/obese women are larger and at higher risk of birth complications. Many maternal obesity-related traits are observationally associated with birth weight, but the causal nature of these associations is uncertain.

Objective-To test for genetic evidence of causal associations of maternal body mass index (BMI) and related traits with birth weight.

Design, Setting and Participants-We used Mendelian randomization to test whether maternal BMI and obesity-related traits are causally related to offspring birth weight. Mendelian

Corresponding authors: Dr. Rachel M. Freathy, University of Exeter Medical School, Royal Devon and Exeter Hospital, Barrack Road Exeter, UK, Tel: +44 (0)1392 408238, r.freathy@ex.ac.uk; Prof. Debbie A. Lawlor, MRC Integrative Epidemiology Unit at the University of Bristol, Oakfield House, Oakfield Road, Bristol, UK, Tel: +44 (0)117 3310096, d.a.lawlor@ bristol.ac.uk; Prof. Timothy M. Frayling, University of Exeter Medical School, Royal Devon and Exeter Hospital, Barrack Road, Exeter, UK, Tel: +44 (0)1392 408256, t.m.frayling@ex.ac.uk.

*These authors jointly directed this work Author Contributions

Dr. Freathy and Profs Lawlor and Frayling had full access to all of the data in the ALSPAC, EFSOCH and HAPO (non-GWAS) studies and access to summary data from all contributing studies and take responsibility for the integrity of the data and accuracy of the data analysis.

Study concept and design: J. Tyrrell, D. A. Lawlor, T. M. Frayling \& R. M. Freathy

Analysis and interpretation of data: J. Tyrrell, R.C. Richmond, S. Metrustry, B. Feenstra, A. Cavadino, E. Hyppönen, M-F Hivert, J.F. Felix, W. L. Lowe, D. A. Lawlor, T. M. Frayling \& R. M. Freathy

Drafting manuscript: J. Tyrrell, R.C. Richmond, S. Metrustry, B. Feenstra, A. Cavadino, E. Hyppönen, M-F Hivert, J.F. Felix, W. L. Lowe, D. A. Lawlor, T. M. Frayling \& R. M. Freathy

Critical revision of manuscript for important intellectual content: All authors

Statistical analysis: N. M. G. De Silva, A. R. Wood, G. McMahon, D. M. Evans, E. Kreiner-M0ller, F. Geller, C. Allard, J-J.

Hottenga, J. P. Bradfield, M. G. Hayes, D. M. Scholtens, L. L. Armstrong, J. Rangaraja, M. Horikoshi, M. I. McCarthy, M. Nodzenski, L. Paternoster, S. Das, V. Huikari, J. N. Painter, S. E. Medland, P. A. Lind, D. J. Berry, R. Myhre, V. Sengpiel, J. Tyrrell, R.C.

Richmond, S. Metrustry, B. Feenstra, A. Cavadino, J.F. Felix, D. A. Lawlor \& R. M. Freathy

Genotyping: D. M. Evans, S. M. Ring, J. C. Murray, L. Bouchard, J. F. Felix, J-J. Hottenga, H. Hakonarson, T. I. A. Sørensen, N. G.

Martin, E. A. Nohr, T. D. Spector, S. F. Grant, D. A. Lawlor, T. M. Frayling \& R. M. Freathy

Individual study design: T. D. Spector, B. Jacobsson, C. Power, N. G. Martin, S. Serbert, T. I. A. Sørensen, M. Jarvelin, B. Hocher, M. G. Hayes, W. L. Lowe, S. F. Grant, H. Hakonarson, J. P. Bradfield, E. J. C. de Geus, V. W. Jaddoe, A. Hofman, M. Melbye, J. C. Murray, H. Bisgaard, G. Davey Smith, A. T. Hattersley, E. Hypponen, M-F Hivert, J.F. Felix, W. L. Lowe, D. A. Lawlor, \& T. M. Frayling

Phenotyping in contributing studies: T. D. Spector, B. Jacobsson, C. Power, E. Hypponen, N. G. Martin, T. I. A. Sørensen, E. A. Nohr, C. Reichetzeder, B. Hocher, S. F. Grant, H. Hakonarson, J. P. Bradfield, G. Willemsen, V. W. Jaddoe, M-F Hivert, M. Melbye, F. Geller, B. Feenstra, E. Kreiner-Møller, H. Bisgaard, G. Davey Smith, S. M. Ring, D. A. Lawlor, B. Knight, A. T. Hattersley

Additional Contributions

We are extremely grateful to the participants and families who contributed to all of the studies and the teams of investigators involved in each one. These include interviewers, computer and laboratory technicians, clerical workers, research scientists, volunteers, managers, receptionists and nurses. For additional study-specific acknowledgements, please see Supplementary Material. This research has been conducted using the UK Biobank resource.

Conflicts of Interest and Financial Disclosures

No conflicts of interest were reported.

Previous Presentations

This work was presented at the Diabetes UK Annual Professional Conference 2014, 5-7 March, Liverpool, UK. 
randomization makes use of the fact that genotypes are randomly determined at conception and are thus not confounded by non-genetic factors. Data were analysed on 30,487 women from 18 studies. Participants were of European ancestry from population- or community-based studies located in Europe, North America or Australia and participating in the Early Growth Genetics (EGG) Consortium. Live, term, singleton offspring born between 1929 and 2013 were included. We tested associations between a genetic score of $30 \mathrm{BMI}$-associated single nucleotide polymorphisms (SNPs) and (i) maternal BMI and (ii) birth weight, to estimate the causal relationship between BMI and birth weight. Analyses were repeated for other obesity-related traits.

Exposures-Genetic scores for BMI, fasting glucose level, type 2 diabetes, systolic blood pressure (SBP), triglyceride level, HDL-cholesterol level, vitamin D status and adiponectin level.

Main Outcome(s) and Measure(s)—Offspring birth weight measured by trained study personnel ( $\mathrm{n}=2$ studies), from medical records ( $\mathrm{n}=10$ studies) or from maternal report $(\mathrm{n}=6$ studies).

Results-Among the 30,487 newborns the mean birth weight in the various cohorts ranged from $3325 \mathrm{~g}$ to $3679 \mathrm{~g}$. The genetic score for BMI was associated with a $2 \mathrm{~g}(95 \% \mathrm{CI}: 0,3 \mathrm{~g}$ ) higher offspring birth weight per maternal BMI-raising allele $(P=0.008)$. The maternal genetic scores for fasting glucose and SBP were also associated with birth weight with effect sizes of $8 \mathrm{~g}$ (95\%CI: 6 , $10 \mathrm{~g})$ per glucose-raising allele $\left(P=7 \times 10^{-14}\right)$ and $-4 \mathrm{~g}(95 \% \mathrm{CI}:-6,-2 \mathrm{~g})$ per SBP-raising allele $\left(P=1 \times 10^{-5}\right)$, respectively. A 1 standard deviation $\left(1 \mathrm{SD} \approx 4 \mathrm{~kg} / \mathrm{m}^{2}\right)$ genetically higher maternal BMI was associated with a $55 \mathrm{~g}(95 \% \mathrm{CI}: 17,93 \mathrm{~g})$ higher birth weight. A 1-SD genetically higher maternal fasting glucose $(\approx 0.4 \mathrm{mmol} / \mathrm{L})$ or SBP $(10 \mathrm{mmHg})$ were associated with a $114 \mathrm{~g}(95 \% \mathrm{CI}$ : $80,147 \mathrm{~g}$ ) higher or $-208 \mathrm{~g}$ (95\% CI: $-394,-21 \mathrm{~g}$ ) lower birth weight, respectively. For BMI and fasting glucose these genetic associations were consistent with the observational associations, but for SBP, the genetic and observational associations were in opposite directions.

Conclusions and Relevance-In this Mendelian randomization study of more than 30,000 women with singleton offspring from 18 studies, genetically elevated maternal BMI and blood glucose levels were potentially causally associated with higher offspring birth weight, whereas genetically elevated maternal systolic blood pressure was shown to be potentially causally related to lower birth weight. If replicated, these findings may have implications for counseling and managing pregnancies to avoid adverse weight-related birth outcomes.

\section{Introduction}

Neonates born to overweight or obese women are more likely to be large for gestational age. ${ }^{1}$ The precise mechanisms underlying this association and the extent to which confounding factors contribute are poorly understood. It is important to understand which maternal traits are causally associated with birth weight because this may (i) facilitate targeted development of interventions to be tested in randomized controlled trials, and (ii) enable clear, evidence-based recommendations in pregnancy.

Maternal overweight and obesity are key risk factors for gestational diabetes. ${ }^{2}$ Even in the absence of diabetes, obese women have higher glucose levels than normal weight women, despite a controlled diet. ${ }^{3}$ The association between gestational diabetes and higher birth 
weight is well documented ${ }^{4}$, and maternal glucose levels below those diagnostic of diabetes also show strong associations with birth weight. ${ }^{5}$

The fetus of an overweight or obese woman may be exposed to the consequences of higher maternal triglyceride levels and blood pressure, lower levels of HDL-cholesterol (HDLc) and adiponectin and lower vitamin D status ${ }^{1,6,7}$ (Box 1). These maternal obesity-related traits have been variably associated with birth weight in observational studies: higher triglycerides and lower HDLc with higher birth weight ${ }^{8,9}$; higher blood pressure with lower birth weight ${ }^{10}$; lower vitamin $\mathrm{D}$ status with lower birth weight ${ }^{11}$; and lower adiponectin with higher birth weight ${ }^{12}$. However, associations are not always consistently observed and may be confounded, for example by maternal socioeconomic status and associated behaviours such as smoking and diet. Furthermore, the high inter-correlation of obesity-related traits complicates determination of causal relationships in an observational setting.

Maternal genotypes may be used in a Mendelian randomization ${ }^{13,14}$ approach to provide evidence of a potential causal association between maternal traits and birth outcomes (Figure 1). Mendelian randomization is analogous to a randomized controlled trial: genotypes, which are randomly allocated at conception, are largely free from confounding and can be used to estimate the possible causal effects of maternal traits. In this study, genetic variants were selected to calculate genetic scores representing maternal BMI and each of 7 obesityrelated maternal traits. The potential causal relationship between maternal BMI and each related trait was estimated by testing associations between maternal genetic risk scores and offspring birth weights.

\section{Methods}

\section{Study participants}

Single nucleotide polymorphism (SNP) genotype data were used from a total of 30,487 women from 18 population- or community-based studies located in Europe, North America or Australia. The birth weight of one child per mother was included (see eTable 1 for full details of participant characteristics and eTable 2 for genotyping information). Birth weight was measured by trained study personnel ( $\mathrm{n}=2$ studies), from medical records ( $\mathrm{n}=10$ studies) or from maternal report ( $\mathrm{n}=6$ studies). The offspring years of birth were from 1929 to 2013. Multiple births, stillbirths, congenital anomalies, births before 37 weeks gestation and individuals of non-European ancestry were excluded. Informed consent was obtained from all participants, and study protocols were approved by the local regional or institutional ethics committees.

\section{Selection of maternal obesity-related traits and SNPs}

In addition to BMI, traits were selected that are associated with maternal obesity and may affect fetal growth through the intrauterine environment. Their effects were modelled in the directions hypothesised by their relationships to maternal BMI (Box 1)

SNPs known to be robustly associated $\left(\mathrm{P}<5 \times 10^{-8}\right)$ with BMI and each obesity-related trait were selected. Full details of the selected SNPs are provided in eTable 3. SNPs associated with (i) fasting glucose and (ii) type 2 diabetes were used to represent maternal glycemia. 
The Type 2 diabetes SNPs were considered to represent exposure to maternal diabetes in pregnancy, including gestational diabetes, given overlap between type 2 and gestational diabetes genetic susceptibility variants. ${ }^{15}$ For blood pressure, SNPs were selected that are primarily associated with systolic blood pressure (SBP), though all also show strong evidence of association with diastolic blood pressure. For vitamin D status, two SNPs with hypothesised roles in vitamin D synthesis were used to represent 25(OH)D levels (an indicator of overall vitamin D status), as previously recommended. ${ }^{16,17}$ Further details of SNP selection are provided in the eMethods.

A weighted genetic score was calculated for each maternal trait (see eMethods for full details). Very few of the selected SNPs have been tested in pregnancy. Genetic scores were validated by confirming that each was associated with its respective maternal trait, measured during pregnancy (with the exception of BMI, for which the pre-pregnancy value was used). Maternal pre-pregnancy BMI was available from registry data ( $\mathrm{N}=2$ studies) or calculated from self-reported weight and height ( $\mathrm{N}=3$ studies). In the Avon Longitudinal Study of Parents and Children (ALSPAC) study, the self-report was validated with a clinic measure ${ }^{18}$. Details of traits measured in pregnancy and their sources are given in eTable 4. In each available study, linear regression of the maternal trait (e.g. BMI) against the genetic score was performed, adjusting for maternal age. To confirm that associations between each genetic score and its respective maternal trait were similar in the same individuals during and after pregnancy, available data were used from two longitudinal studies (the Avon Longitudinal Study of Parents and Children [ALSPAC] and the Exeter Family Study of Childhood Health [EFSOCH]). To check that the strategy for SNP selection had resulted in genetic scores that were specific to each maternal trait, the association was tested between each of the 8 genetic scores and the other maternal traits, and indicators of maternal socioeconomic status and smoking.

\section{Testing the hypothesis that maternal BMI and obesity-related traits are associated with birth weight through the intra-uterine environment}

For BMI and each related maternal trait, two Mendelian randomization approaches were used to test the hypothesis. First, associations were tested between genetic scores representing maternal traits and offspring birth weight using the maximum number of participants (i.e. for each trait, those with genetic score and offspring birth weight data available, irrespective of whether they had the maternal trait measured). An association of the genetic score with birth weight would support a possible causal relationship between the trait (e.g. pre-pregnancy BMI) and birth weight, but would not provide information on the size of that association. Second, we performed analyses in those with the measured trait that enabled an estimate of the size of a possible causal relationship. The analyses took into account the association between each genetic score and the maternal trait it represented (e.g. BMI), in addition to the association between the same genetic score and birth weight. These two results were used to calculate an association between the maternal trait (e.g. BMI) and birth weight that was free from confounding. This second approach measures the relationship between variation in maternal BMI (or BMI-related trait) and birth weight that is attributable only to genetic factors (see Figure 1 for an explanation of the method). For 
each approach meta-analysis was used to combine data from individual studies (see eMethods).

Using the first approach, we investigated the association between each genetic score and (i) birth weight and (ii) ponderal index (an index of neonatal leanness, measured in $\mathrm{kg} / \mathrm{m}^{3}$ ). With in each study, birth weight or ponderal index $Z$-scores were regressed against each maternal genetic score, adjusted for offspring sex and gestational age. Analyses using the type 2 diabetes genetic score were repeated after excluding participants with pre-existing and gestational diabetes. Analyses using the SBP genetic score were repeated after excluding participants with pre-eclampsia and existing or gestational hypertension.

The genetic estimate of the association between each maternal trait and birth weight/ ponderal index from the second approach was compared with the corresponding observational association. To obtain the observational estimates linear regression was performed using birth weight or ponderal index as the dependent variable, and each of 7 maternal traits as independent variables, adjusting for sex and gestational age. There was insufficient information on maternal type 2 diabetes prevalence, so it was not possible to estimate the causal relationship for that trait. Full details of the analysis are provided in the eMethods.

\section{Estimating how much of the association between maternal BMI and birth weight is mediated by fasting glucose}

Available data were used to estimate the approximate causal relationship between a $1 \mathrm{SD}(\approx$ $4 \mathrm{~kg} / \mathrm{m}^{2}$ ) higher maternal BMI and (i) fasting glucose and (ii) SBP. Using each of those estimates, the results of the Mendelian randomization analyses were rescaled to represent the effects of fasting glucose and SBP that could be directly compared with the causal relationship between a 1 SD higher BMI and birth weight (see eMethods for a detailed description of the method).

\section{Correcting for direct fetal genotype effects}

Genotypes of maternal-fetal pairs were available in up to 8 studies ( $\mathrm{N}=$ up to 11,494). Analyses were repeated including the fetal genotype at each SNP in the model, to correct for potential confounding caused by direct effects of the fetal genotype.

\section{Results}

The characteristics of included participants from the 18 contributing studies are shown in Table 1. Among the 30,487 newborns the mean birth weight ranged from $3325 \mathrm{~g}$ to $3679 \mathrm{~g}$. The mean prepregnancy BMI was available in 11 studies and ranged from $22.78 \mathrm{~kg} / \mathrm{m}^{2}$ to $24.83 \mathrm{~kg} / \mathrm{m}^{2}$. The mean maternal age at delivery, available in 16 studies, ranged from 24.5 years to 31.5 years.

There was evidence of association between each genetic score and its corresponding maternal trait measured in pregnancy $(P \unlhd 0.003$; Table 2). For BMI, fasting glucose and SBP, data from multiple studies were meta-analysed, with similar effect estimates between studies for BMI and fasting glucose $\left(P_{h e t}>0.05\right)$ and evidence of heterogeneity for $\operatorname{SBP}\left(P_{h e}=0.04\right)$. 
The effect sizes of associations between maternal traits and their respective genetic scores were very similar when compared in the same individuals during and outside pregnancy, with the exception of the SBP genetic score which had a weaker effect during pregnancy (eTable 5). There was no evidence of association between any genetic score and potentially confounding variables. No individual genetic score was associated with any of the other maternal traits, except for the genetic score for BMI, which was positively associated with SBP $(P<0.003$ Bonferroni-corrected for 15 tests; eTable 6$)$.

\section{Genetic evidence for a possible causal association between higher maternal BMI and higher birth weight}

The maternal BMI genetic score was associated with higher birth weight (Table 3) and ponderal index (eTable 7) with similar effect sizes before and after adjusting for possible effects of fetal genotype. Using the genetic score to quantify the possible causal association, a 1 SD genetically higher maternal BMI (equivalent to $4 \mathrm{~kg} / \mathrm{m}^{2}$ ) was associated with a $55 \mathrm{~g}$ (95\%CI: 17, 93) higher offspring birth weight. After adjusting for fetal genotype, the estimated effect was 104g (95\%CI: 32, 176) (Table 4). These Mendelian randomization causal estimates were similar to the observational association of $62 \mathrm{~g}(95 \% \mathrm{CI}: 56,70)$ per $1 \mathrm{SD}\left(4 \mathrm{~kg} / \mathrm{m}^{2}\right)$ higher maternal BMI (Figure 2). Similar results were obtained for ponderal index (eTable 8 and eFigure 1).

\section{Genetic evidence for a possible causal association between higher maternal fasting glucose and higher birth weight, but no association with maternal lipids or adiponectin}

The maternal fasting glucose and type 2 diabetes genetic scores were associated with higher birth weight (Table 3) and ponderal index (eTable 7) with similar effect size estimates before and after adjusting for fetal genotype, and before and after excluding pre-existing and gestational diabetes. Using the genetic score to estimate the possible causal relationship, a $1 \mathrm{SD}(0.4 \mathrm{mmol} / \mathrm{L})$ genetically higher maternal glucose was associated with a $114 \mathrm{~g}(95 \% \mathrm{CI}$ : $80,147)$ higher birth weight. After adjusting for fetal genotype, the association was $145 \mathrm{~g}$ (95\%CI: 91, 199) (Table 4). These genetic estimates were similar to the observational association of $92 \mathrm{~g}(95 \% \mathrm{CI}: 80,104)$ per $1 \mathrm{SD}(0.4 \mathrm{mmol} / \mathrm{L})$ higher maternal glucose (Figure 2). Similar results were obtained for ponderal index (eTable 8 and eFigure 1).

The maternal triglyceride genetic score was not associated with offspring birth weight (Table 3) or ponderal index (eTable 7). Using the genetic score to estimate the possible causal relationship, a genetically higher maternal triglyceride level was not associated with offspring birth weight and the $95 \%$ confidence intervals around the genetic estimate excluded the observational association between maternal triglycerides and birth weight ( $P=0.007$ testing difference between genetic and observational association; Table 4; Figure 2). Likewise, the genetic estimate of the possible effect of maternal adiponectin levels on offspring birth weight was different from the observational association $(P=0.002)$. The genetic score for HDLc was not associated with birth weight or ponderal index and the analysis was consistent with no causal relationship, however this could not be distinguished from the negative observational association between maternal HDLc and birth weight. 


\section{Genetic evidence for a possible causal association between higher systolic blood pressure and lower birth weight}

The maternal SBP genetic score was associated with lower birth weight (Table 3) and ponderal index (eTable 7) with similar effect size estimates before and after adjusting for fetal genotype, and before and after excluding maternal pre-eclampsia and hypertension. Using the genetic score to estimate the possible causal relationship, a $1 \mathrm{SD}(10 \mathrm{mmHg})$ genetically higher maternal SBP was associated with a $-208 \mathrm{~g}(95 \% \mathrm{CI} ;-394,-21)$ lower offspring birth weight. After adjusting for fetal genotype, the estimated effect was $-151 \mathrm{~g}$ (95\%CI: $-390,89)$ (Table 4). The genetic estimate of the association between maternal SBP and birth weight in the full sample of women was in the opposite direction to the observational association ( $P=0.01$ for difference between genetic and observational associations; Table 4; Figure 2). Similar results were obtained for ponderal index (eTable 8 and eFigure 1).

The maternal genetic score for lower vitamin $\mathrm{D}$ status was associated with lower birth weight ( $P=0.03$; Table 3 ). However, the estimated causal relationship was not significantly different from zero (the estimated change in birth weight for a $10 \%$ genetically lower maternal 25[OH]D level was $-26 \mathrm{~g}$ (95\%CI: $-54,2)$; Table 4, Figure 2).

\section{Associations between the genetic scores and birth weight were consistent across studies}

Associations between maternal genetic scores and offspring birth weight were similar between studies in the meta-analysis (Table $3 ; P_{h e t}>0.05$ ). Where data were combined from observational analyses, the associations between maternal fasting glucose or SBP and birth weight were similar $\left(P_{h e t}>0.05\right)$, and there was evidence of heterogeneity for the BMI-birth weight observational association (Table $4 ; P_{h e}=0.03$ ).

\section{Exposure of the fetus to higher maternal fasting glucose is unlikely to explain all of the association between higher maternal BMI and higher offspring birth weight}

To estimate how much of the association between maternal BMI and birth weight might be mediated by fasting glucose, the BMI and fasting glucose genetic scores were used: a $1 \mathrm{SD}$ $\left(\approx 4 \mathrm{~kg} / \mathrm{m}^{2}\right)$ genetically higher maternal BMI was associated with a $0.34 \mathrm{SD}(\approx 0.14$ $\mathrm{mmol} / \mathrm{L}$ ) higher maternal fasting glucose. From the Mendelian randomization analyses, 1 $\mathrm{SD}(\approx 0.4 \mathrm{mmol} / \mathrm{L})$ genetically higher maternal fasting glucose was associated with a $114 \mathrm{~g}$ (95\%CI: 80,147) higher birth weight. Consequently, it was predicted that a 0.34 SD higher fasting glucose would be associated with a $114 \mathrm{~g} \times 0.34=39 \mathrm{~g}$ [95\%CI: 27, 50] higher birth weight. This approximation is broadly similar to the total estimated effect of a $1 \mathrm{SD}$ higher BMI on birth weight $(55 \mathrm{~g}$ [95\% CI: 17, 93]). However, using the same method with the BMI and SBP genetic scores we estimated that a 1SD higher maternal BMI would be associated with a $-40 \mathrm{~g}$ [95\%CI:-75, -4] lower birth weight via its association with maternal SBP (eFigure 2), which would oppose the positive association with maternal fasting glucose.

\section{Discussion}

This study provides evidence for a possible causal association between maternal BMI and offspring birth weight. A $4 \mathrm{~kg} / \mathrm{m}^{2}$ genetically higher maternal BMI (a 1 SD rise) was 
associated with a 55g (95\% CI: 17, 93) higher offspring birth weight. In addition, a 0.4 $\mathrm{mmol} / \mathrm{l}(1 \mathrm{SD})$ genetically higher circulating maternal fasting glucose was associated with a $114 \mathrm{~g}(95 \% \mathrm{CI}: 80,147)$ higher birth weight, while a $10 \mathrm{mmHg}$ genetically higher maternal SBP was associated with a $-208 \mathrm{~g}(95 \% \mathrm{CI}:-394,-21)$ lower birth weight. These results provide evidence that genetically elevated maternal glucose and SBP have directionally opposite causal associations with birth weight. The estimated associations between these maternal traits and birth weight (either increased or reduced) are substantial and of clinical importance. They support efforts to maintain healthy gestational glucose and blood pressure levels to ensure healthy fetal growth. The positive association between maternal BMI and birth weight may be partially mediated by the effect of higher BMI on circulating maternal fasting glucose. There was no evidence of association with a genetic score for maternal triglycerides, which have also been hypothesised to be important contributors to higher birth weight in overweight or obese women. Other lipids, or specific subclasses of triglycerides, might be important but require further study.

These results provide genetic evidence of a causal association between maternal glycemia and birth weight and ponderal index, even in women with no pre-existing or gestational diabetes, which is consistent with published observational data. ${ }^{5}$ A possible explanation for this finding is that women with a higher genetic score for type 2 diabetes have relatively higher glucose levels in pregnancy, as a result of inadequate beta cell compensation in response to gestational insulin resistance, ${ }^{19,20}$ leading to increased placental glucose transfer and fetal insulin secretion, ${ }^{21}$ and consequently higher birth weight.

These data did not support a causal association between maternal triglyceride, HDLc or adiponectin levels and birth weight or ponderal index. The genetic associations between maternal triglycerides and adiponectin and birth weight were null, in contrast to the observational associations, suggesting that the observational associations seen here, and in other published studies ${ }^{8,9,12}$, are confounded.

The Mendelian randomization analysis showed that the positive observational association between SBP and birth weight is confounded, most likely by BMI, which is both an important risk factor for higher SBP in pregnancy and positively associated with birth weight. ${ }^{1}$ Using genetic variants that are independent of confounding by BMI, it was demonstrated that genetically higher maternal SBP is associated with lower birth weight, even after excluding pre-eclampsia and hypertension. The precision of our estimate of the change in birth weight per 1 SD in maternal SBP could be affected by the heterogeneity between studies in the genetic score-SBP association ( $P=0.04, P^{2}=76.0 \%$; Table 2$)$. However, associations between the SBP genetic score and birth weight were consistent across all 13 meta-analyzed studies ( $P=0.14, I^{2}=30.4 \%$; Table 3 ) and supportive of a causal association between higher maternal SBP and lower birth weight. These findings support observational associations between maternal SBP and birth weight that were adjusted for a wide range of confounders, ${ }^{22}$ and are consistent with laboratory and population studies suggesting a link between hypertensive disorders of pregnancy and impaired fetal growth due to placental pathology. ${ }^{23}$ There are increasing concerns about the effect the obesity epidemic might have on birth size, via greater maternal BMI. However, the focus of that concern has been largely 
on increased birth size as a result of greater maternal glucose and other fetal nutrients. Our findings suggest that there are opposing effects of maternal blood pressure and glucose.

Published Mendelian randomization analyses provide evidence that higher BMI is causally associated with lower vitamin D status, ${ }^{6}$ and evidence from multiple observational studies suggests that lower maternal vitamin $\mathrm{D}$ is associated with lower birth weight. ${ }^{11,24}$ Our analysis of the vitamin D genetic score provided some evidence to support a possible causal association with birth weight, but this requires further exploration in larger numbers of pregnancies.

Socio-economic factors and related behaviours such as smoking are key confounders of observational associations between maternal BMI (or BMI-related traits) and offspring birth weight, since they are associated with both variables (see eTable 9 for a demonstration of these associations in the ALSPAC study). The genetic scores used in our analyses were not associated with socioeconomic factors or smoking, and this illustrates a key strength of the Mendelian randomization approach: since genotypes are determined at conception, such confounding is avoided.

There are some limitations to this study. Despite attempts to maximise specificity of the genetic scores, we cannot fully exclude the possibility that the selected genetic variants act on more than one maternal trait. Although all available information was used, there was limited power to detect associations between the genetic scores and other traits. For example, the known association between BMI-associated variants and triglyceride levels was not detected. ${ }^{25}$ With the potential for high-throughput metabolomic studies and a growing public database of genetic associations, ${ }^{26-28}$ future studies will improve the specificity (for different lipid sub-fractions) of selected genetic variants.

Despite the large sample in this study, statistical power to detect causal relationships was limited for some maternal traits (see eMethods and eTable 10 for power calculations). The total sample provided $>99 \%$ power to detect associations at $\mathrm{P}<0.05$ between birth weight and genetic scores such as fasting glucose and systolic blood pressure that explain at least $0.1 \%$ variance in birth weight. However, larger samples $(\mathrm{N}>80,000)$ will be needed to confidently detect or rule out (i) the association with vitamin D status suggested by our data, or (ii) smaller positive or negative causal associations between maternal triglycerides, HDLc or adiponectin and birth weight.

While adjusting for the fetal genetic scores was necessary to separate maternal effects from the direct effects of genetic variants in the fetus, this could potentially introduce bias via association with paternal genotypes. Assortative mating for BMI could additionally result in a correlation between maternal and paternal genotypes, leading to similar bias. However, a father's genetic score would only confound the Mendelian randomization estimates if the father's phenotype were related to birth weight, and we found only very weak associations of fathers BMI and related traits with offspring birth weight (eTable 11). Another potential bias could be induced by the use of the genetic score for SBP, which was derived from a genome-wide association study of blood pressure conditional on BMI. Since BMI is also 
associated with birth weight, this could bias the results. However, similar results were obtained using an alternative genetic score that was unadjusted for BMI (eMethods).

In Mendelian randomization analysis, a weak statistical association between a genetic score and a maternal trait (due to low variance explained and/or small sample size) has the potential to cause weak instrument bias towards the observational results. ${ }^{29}$ The proportions of maternal trait variance explained by the genetic scores are modest in our study (Table 2). However, the large overall sample size ensured that the possible causal associations identified are unlikely to be due to weak instrument bias (see eMethods).

Our analyses assume that maternal BMI and related traits are linearly associated with offspring birth weight. We have not tested for non-linear associations which, in a Mendelian randomization design, would require very large numbers ${ }^{30}$. H owever, for maternal BMI, fasting glucose and systolic blood pressure, there is observational evidence of such linear associations across the distribution, with no evidence of threshold or curvilinear associations ${ }^{5,10,31}$.

\section{Conclusions}

In this Mendelian randomization study of more than 30,000 women with singleton offspring from 18 studies, genetically elevated maternal BMI and blood glucose levels were potentially causally associated with higher offspring birth weight, whereas genetically elevated maternal systolic blood pressure was shown to be potentially causally related to lower birth weight. If replicated, these findings may have implications for counseling and managing pregnancies to avoid adverse weight-related birth outcomes.

\section{Maternal traits hypothesized to increase fetal growth:}

- Higher BMI

- Higher fasting glucose

- Gestational or Type 2 diabetes

- Higher triglycerides

- Lower HDL-cholesterol

- Lower adiponectin

Maternal traits hypothesized to decrease fetal growth:

- Higher blood pressure

- Lower vitamin D status

Box 1.

The maternal obesity-related traits hypothesized to cause increased or decreased fetal growth, based on observational associations with birth weight: body mass index (BMI) ${ }^{1}$; fasting glucose ${ }^{5}$; gestational or type 2 diabetes $^{32}$; triglycerides $^{9}$; HDL-cholesterol ${ }^{8}$; 
systolic blood pressure ${ }^{10}$; vitamin D status (as indicated by 25 -hydroxyvitamin $\mathrm{D}$, 25[OH]D level) ${ }^{11}$; adiponectin ${ }^{12}$.

\section{Supplementary Material}

Refer to Web version on PubMed Central for supplementary material.

\section{Authors}

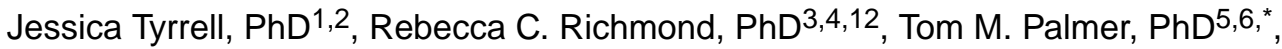
Bjarke Feenstra, $\mathrm{PhD}^{7}$, Janani Rangarajan, $\mathrm{MS}^{8}$, Sarah Metrustry, $\mathrm{MSc}^{9}$, Alana Cavadino, MSc ${ }^{10,11}$, Lavinia Paternoster, $\mathrm{PhD}^{12}$, Loren L. Armstrong, $\mathrm{PhD}^{13}, \mathrm{~N}$. Maneka G. De Silva, PhD ${ }^{12}$, Andrew R. Wood, PhD1, Momoko Horikoshi, MD, $\mathrm{PhD}^{14,15}$, Frank Geller, $\mathrm{MSc}^{7}$, Ronny Myhre, $\mathrm{PhD}^{16}$, Jonathan P. Bradfield, BS ${ }^{17}$, Eskil Kreiner-Møller, $\mathrm{MD}^{18}$, Ville Huikari, $\mathrm{MSc}^{19}$, Jodie N. Painter, $\mathrm{PhD}^{20}$, Jouke-Jan Hottenga, $\mathrm{PhD}^{21,22}$, Catherine Allard, BSc ${ }^{23,24}$, Diane J. Berry, $\mathrm{PhD}^{11}$, Luigi Bouchard, PhD, MBA ${ }^{24,25,26}$, Shikta Das, PhD 27 , David M. Evans, $\mathrm{PhD}^{3,12,28}$, Hakon Hakonarson, MD, PhD ${ }^{17,29,30}$, M. Geoffrey Hayes, $\mathrm{PhD}^{13}$, Jani Heikkinen, $\mathrm{MSc}^{31}$, Albert Hofman, $\mathrm{PhD}^{32}$, Bridget Knight, $\mathrm{PhD}^{1}$, Penelope A. Lind, $\mathrm{PhD}^{20}$, Mark I. McCarthy, MD, $\mathrm{PhD}^{14,15,33}$, George McMahon, $\mathrm{PhD}^{3}$, Sarah E. Medland, $\mathrm{PhD}^{20}$, Mads Melbye, MD, DMSc ${ }^{7,34}$, Andrew P. Morris, PhD ${ }^{15,35}$, Michael Nodzenski, $\mathrm{MS}^{8}$, Christoph Reichetzeder, $\mathrm{MD}^{36,37}$, Susan M. Ring, $\mathrm{PhD}^{3,12}$, Sylvain Sebert, $P h D^{19,38}$, Verena Sengpiel, $P h D^{39}$, Thorkild I.A. Sørensen, MD ${ }^{12,40,41}$, Gonneke Willemsen, $\mathrm{PhD}^{21,22}$, Eco J. C. de Geus, $\mathrm{PhD}^{21,22}$, Nicholas G. Martin, $\mathrm{PhD}^{20}$, Tim D. Spector, $\mathrm{MD}^{9}$, Christine Power, $\mathrm{PhD}^{11}$, Marjo-Riitta Järvelin, $\mathrm{MD}$, $\mathrm{PhD}^{19,38,42,43,44}$, Hans Bisgaard, MD, DMSci ${ }^{18}$, Struan F.A. Grant, PhD 17,29,30, Ellen A. Nohr, PhD ${ }^{45}$, Vincent W. Jaddoe, $\mathrm{PhD}^{4,32,46}$, Bo Jacobsson, MD, PhD ${ }^{16,39}$, Jeffrey C. Murray, $\mathrm{MD}^{47}$, Berthold Hocher, MD, $\mathrm{PhD}^{36,48}$, Andrew T. Hattersley, $\mathrm{DM}^{1}$, Denise M. Scholtens, $\mathrm{PhD}^{8}$, George Davey Smith, $\mathrm{DSc}^{3,12}$, Marie-France Hivert, $\mathrm{MD}^{49,50,51}$, Janine F. Felix, $\mathrm{PhD}^{4,32,46}$, Elina Hyppönen, $\mathrm{PhD}^{11,52,53}$, William

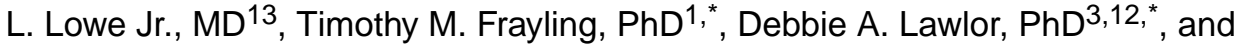

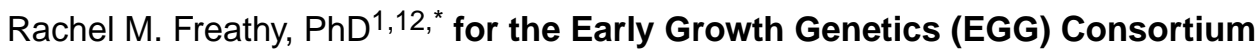

\section{Affiliations}

${ }^{1}$ Institute of Biomedical and Clinical Science, University of Exeter Medical School, Royal Devon and Exeter Hospital, Barrack Road, Exeter, EX2 5DW, UK ²European Centre for Environment and Human Health, University of Exeter, The Knowledge Spa, Truro, TR1 3HD ${ }^{3}$ School of Social and Community Medicine, University of Bristol, Oakfield House, Oakfield Grove, Bristol, BS8 2BN, UK ${ }^{4}$ The Generation R Study Group, Erasmus MC, University Medical Center Rotterdam, P.O.Box 2040, 3000 CA, Rotterdam, the Netherlands ${ }^{5}$ Division of Health Sciences, Warwick Medical School, University of Warwick, Coventry, UK ${ }^{6}$ Department of Mathematics and Statistics, Lancaster University, Lancaster, UK ${ }^{7}$ Department of Epidemiology Research, Statens Serum Institut, Copenhagen, Denmark ${ }^{8}$ Department of Preventive Medicine, Northwestern University Feinberg School of Medicine 
'Department of Twin Research, King's College London, St. Thomas' Hospital, London, UK ${ }^{10}$ Centre for Environmental and Preventive Medicine, Wolfson Institute of Preventive Medicine, Barts and the London School of Medicine and Dentistry, Queen Mary University of London ${ }^{11}$ Population, Policy and Practice, UCL Institute of Child Health, University College London, UK ${ }^{12}$ Medical Research Council Integrative Epidemiology Unit at the University of Bristol, UK ${ }^{13}$ Division of Endocrinology, Metabolism and Molecular Medicine, Northwestern University Feinberg School of Medicine ${ }^{14}$ Oxford Centre for Diabetes, Endocrinology and Metabolism, University of Oxford, UK ${ }^{15}$ Wellcome Trust Centre for Human Genetics, University of Oxford, Oxford, UK ${ }^{16}$ Department of Genes and Environment, Division of Epidemiology, Norwegian Institute of Public Health, Oslo, Norway ${ }^{17}$ Center for Applied Genomics, The Children's Hospital of Philadelphia, Philadelphia, Pennsylvania, USA ${ }^{18}$ Copenhagen Prospective Studies on Asthma in Childhood (COPSAC), Faculty of Health Sciences, University of Copenhagen, Copenhagen, Denmark \& Danish Pediatric Asthma Center, Copenhagen University Hospital, Gentofte, Denmark ${ }^{19}$ Institute of Health Sciences, University of Oulu, Oulu, Finland ${ }^{20}$ QIMR Berghofer Medical Research Institute, Locked Bag 2000, Royal Brisbane Hospital, Herston, Qld 4029, Australia ${ }^{21}$ EMGO Institute for Health and Care Research, VU University Medical Center, Amsterdam, The Netherlands ${ }^{22}$ Department of Biological Psychology, VU University Amsterdam, Van der Boechorststraat 1, 1081 BT Amsterdam, The Netherlands ${ }^{23}$ Department of Mathematics, Universite de Sherbrooke, QC, Canada ${ }^{24}$ Centre de recherche du Centre Hospitalier Universitaire de Sherbrooke, Sherbrooke, QC, Canada ${ }^{25}$ ECOGENE-21 and Lipid Clinic, Chicoutimi Hospital, Saguenay, QC, Canada ${ }^{26}$ Department of Biochemistry, Université de Sherbrooke, Sherbrooke, QC, Canada ${ }^{27}$ Department of Primary Care and Public Health, Imperial College London ${ }^{28}$ University of Queensland Diamantina Institute, Translational Research Institute, Brisbane, Queensland, Australia ${ }^{29}$ Division of Human Genetics, The Children's Hospital of Philadelphia, Philadelphia, Pennsylvania, USA ${ }^{30}$ Department of Pediatrics, Perelman School of Medicine, University of Pennsylvania, Philadelphia, Pennsylvania, USA ${ }^{31}$ FIMM Institute for Molecular Medicine Finland, Helsinki University Helsinki, FI-00014, Finland ${ }^{32}$ Department of Epidemiology, Erasmus MC, University Medical Center Rotterdam, P.O.Box 2040, 3000 CA, Rotterdam, the Netherlands ${ }^{33}$ Oxford National Institute for Health Research (NIHR) Biomedical Research Centre, Churchill Hospital, Oxford, UK ${ }^{34}$ Department of Medicine, Stanford University School of Medicine, Stanford, California, USA ${ }^{35}$ Department of Biostatistics, University of Liverpool, Liverpool L69 3GA, UK ${ }^{36}$ Institute of Nutritional Science, University of Potsdam, Germany ${ }^{37}$ Center for Cardiovascular Research / Charité, Berlin, Germany ${ }^{38}$ Department of Epidemiology and Biostatistics, School of Public Health, Medical Research CouncilHealth Protection Agency Centre for Environment and Health, Faculty of Medicine, Imperial College London, UK ${ }^{39}$ Department of Obstetrics and Gynecology, Sahlgrenska Academy, Sahgrenska University Hospital, Gothenburg, Sweden ${ }^{40}$ Institute of Preventive Medicine, Bispebjerg and Frederiksberg University Hospital, Capital Region, Copenhagen, Denmark ${ }^{41}$ Novo Nordisk Foundation Center for Basic 
Metabolic Research and Department of Public Health, Faculty of Health and Medical Sciences, University of Copenhagen, Copenhagen, Denmark ${ }^{42}$ Department of Children and Young People and Families, National Institute for Health and Welfare, Aapistie 1, Box 310, Fl-90101 Oulu, Finland ${ }^{43}$ Biocenter Oulu, University of Oulu, Oulu, Finland ${ }^{44}$ Unit of Primary Care, Oulu University Hospital, Kajaanintie 50, P.O.Box 20, FI-90220 Oulu, 90029 OYS, Finland ${ }^{45}$ Research Unit of Obstetrics \& Gynecology, Institute of Clinical Research, University of Southern Denmark, Odense, Denmark ${ }^{46}$ Department of Pediatrics, Erasmus MC, University Medical Center Rotterdam, P.O.Box 2040, 3000 CA, Rotterdam, the Netherlands ${ }^{47}$ Department of Pediatrics, University of lowa, lowa City, lowa, USA ${ }^{48}$ The First Affiliated Hospital of Jinan University, Guangzhou, 510630, China ${ }^{49}$ Department of Population Medicine, Harvard Pilgrim Health Care Institute, Harvard Medical School, Boston, MA ${ }^{50}$ Diabetes Center, Massachussetts General Hospital, Boston, MA ${ }^{51}$ Department of Medicine, Universite de Sherbrooke, QC, Canada ${ }^{52}$ Centre for Population Health Research, School of Health Sciences, and Sansom Institute, University of South Australia, Adelaide, Australia ${ }^{53}$ South Australian Health and Medical Research Institute, Adelaide, Australia

\section{Acknowledgments}

\section{Funding/Support}

Funding/support of authors is as follows (funding details for individual studies are reported in the Supplementary Material). T.M.F and A.R.W are supported by the European Research Council grant: 323195 SZ-245 50371GLUCOSEGENES-FP7-IDEAS-ERC; A.T.H. and M.I.M. are Wellcome Trust Senior Investigators; M.I.M. is an NIHR Senior Investigator. R.M.F. is a Sir Henry Dale Fellow (Wellcome Trust and Royal Society grant: 104150/Z/14/Z); J.T. is funded by the ERDF and a Diabetes Research and Wellness Foundation Fellowship; RCR is funded by the Wellcome Trust 4-year studentship (Grant Code: WT083431MF). D.A.L., G.D-S., D.M.E. and S.R. all work in a Unit that receives funding from the University of Bristol and the UK Medical Research Council ( MC_UU_1201/1/5, MC_UU_1201/1 and MC_UU_1201/4). D.A.L. is supported by awards from the Wellcome Trust (WT094529MA and WT088806), US National Institute of Health (R01 DK10324) and is an NIHR Senior Investigator (NF-SI-0611-10196). D.M.E and S.E.M. were supported by Australian Research Council Future Fellowship (FT130101709 and FT110100548). B.F is supported by an Oak Foundation Scholarship. L.B. is a junior research scholar from the Fonds de la recherche en santé du Québec (FRQS) and a member of the FRQS-funded Centre de recherche du CHUS. M.F.H. is a FRQS research scholars and was awarded a Clinical Scientist Award by the Canadian Diabetes Association and the Maud Menten Award from the Institute of Genetics-Canadian Institute of Health Research (CIHR). C.A. was awarded the CIHR - Frederick Banting and Charles Best Canada Graduate Scholarships. V.W.J. is supported by the Netherlands Organization for Health Research and Development (ZonMwVIDI 016.136.361). A.P.M. is a Wellcome Trust Senior Research Fellow (grant number WT098017). T.S. is holder of an ERC Advanced Principal Investigator award.

\section{Role of the Sponsors}

The funding agencies had no role in the design and conduct of the study; collection, management, analysis and interpretation of data; preparation, review, approval of manuscript; or decision to submit manuscript for publication.

\section{REFERENCES}

1. Lawlor DA, Relton C, Sattar N, Nelson SM. Maternal adiposity--a determinant of perinatal and offspring outcomes? Nat Rev Endocrinol. 2012; 8(11):679-688. [PubMed: 23007319]

2. Shin D, Song WO. Prepregnancy body mass index is an independent risk factor for gestational hypertension, gestational diabetes, preterm labor, and small- and large-for-gestational-age infants. J Matern Fetal Neonatal Med. 2014:1-8. 
3. Harmon KA, Gerard L, Jensen DR, et al. Continuous glucose profiles in obese and normal-weight pregnant women on a controlled diet: metabolic determinants of fetal growth. Diabetes Care. 2011; 34(10):2198-2204. [PubMed: 21775754]

4. Landon MB, Mele L, Spong CY, et al. The relationship between maternal glycemia and perinatal outcome. Obstet Gynecol. 2011; 117(2 Pt 1):218-224. [PubMed: 21309194]

5. Metzger BE, Lowe LP, Dyer AR, et al. Hyperglycemia and adverse pregnancy outcomes. N Engl J Med. 2008; 358(19):1991-2002. [PubMed: 18463375]

6. Vimaleswaran KS, Berry DJ, Lu C, et al. Causal relationship between obesity and vitamin D status: bi-directional Mendelian randomization analysis of multiple cohorts. PLoS Med. 2013; 10(2):e1001383. [PubMed: 23393431]

7. Gaillard R, Durmus B, Hofman A, Mackenbach JP, Steegers EA, Jaddoe VW. Risk factors and outcomes of maternal obesity and excessive weight gain during pregnancy. Obesity (Silver Spring). 2013; 21(5):1046-1055. [PubMed: 23784909]

8. Misra VK, Trudeau S, Perni U. Maternal serum lipids during pregnancy and infant birth weight: the influence of prepregnancy BMI. Obesity (Silver Spring). 2011; 19(7):1476-1481. [PubMed: 21394096]

9. Kulkarni SR, Kumaran K, Rao SR, et al. Maternal lipids are as important as glucose for fetal growth: findings from the Pune Maternal Nutrition Study. Diabetes Care. 2013; 36(9):2706-2713. [PubMed: 23757425]

10. Macdonald-Wallis C, Tilling K, Fraser A, Nelson SM, Lawlor DA. Associations of blood pressure change in pregnancy with fetal growth and gestational age at delivery: findings from a prospective cohort. Hypertension. 2014; 64(1):36-44. [PubMed: 24821945]

11. Leffelaar ER, Vrijkotte TG, van Eijsden M. Maternal early pregnancy vitamin D status in relation to fetal and neonatal growth: results of the multi-ethnic Amsterdam Born Children and their Development cohort. Br J Nutr. 2010; 104(1):108-117. [PubMed: 20193097]

12. Lowe LP, Metzger BE, Lowe WL Jr. et al. Inflammatory mediators and glucose in pregnancy: results from a subset of the Hyperglycemia and Adverse Pregnancy Outcome (HAPO) Study. J Clin Endocrinol Metab. 2010; 95(12):5427-5434. [PubMed: 20843942]

13. Davey Smith G, Ebrahim S. 'Mendelian randomization': can genetic epidemiology contribute to understanding environmental determinants of disease? Int J Epidemiol. 2003; 32(1):1-22. [PubMed: 12689998]

14. Lawlor DA, Harbord RM, Sterne JA, Timpson N, Davey Smith G. Mendelian randomization: using genes as instruments for making causal inferences in epidemiology. Stat Med. 2008; 27(8):11331163. [PubMed: 17886233]

15. Kwak SH, Kim SH, Cho YM, et al. A genome-wide association study of gestational diabetes mellitus in Korean women. Diabetes. 2012; 61(2):531-541. [PubMed: 22233651]

16. Berry DJ, Vimaleswaran KS, Whittaker JC, Hingorani AD, Hypponen E. Evaluation of genetic markers as instruments for Mendelian randomization studies on vitamin D. PLoS One. 2012; 7(5):e37465. [PubMed: 22629401]

17. Vimaleswaran KS, Cavadino A, Berry DJ, et al. Association of vitamin D status with arterial blood pressure and hypertension risk: a mendelian randomisation study. Lancet Diabetes Endocrinol. 2014; 2(9):719-729. [PubMed: 24974252]

18. Lawlor DA, Fraser A, Lindsay RS, et al. Association of existing diabetes, gestational diabetes and glycosuria in pregnancy with macrosomia and offspring body mass index, waist and fat mass in later childhood: findings from a prospective pregnancy cohort. Diabetologia. 2010; 53(1):89-97. [PubMed: 19841891]

19. Hayes MG, Urbanek M, Hivert MF, et al. Identification of HKDC1 and BACE2 as genes influencing glycemic traits during pregnancy through genome-wide association studies. Diabetes. 2013; 62(9):3282-3291. [PubMed: 23903356]

20. Freathy RM, Hayes MG, Urbanek M, et al. Hyperglycemia and Adverse Pregnancy Outcome (HAPO) study: common genetic variants in GCK and TCF7L2 are associated with fasting and postchallenge glucose levels in pregnancy and with the new consensus definition of gestational diabetes mellitus from the International Association of Diabetes and Pregnancy Study Groups. Diabetes. 2010; 59(10):2682-2689. [PubMed: 20682688] 
21. Pedersen, J. The pregnant diabetic and her newborn: problems and management. Williams \& Wilkins; Baltimore: 1977.

22. Lawlor DA, Davey Smith G, Ebrahim S. Birth weight of offspring and insulin resistance in late adulthood: cross sectional survey. BMJ. 2002; 325(7360):359. [PubMed: 12183306]

23. Rich-Edwards JW, Fraser A, Lawlor DA, Catov JM. Pregnancy characteristics and women's future cardiovascular health: an underused opportunity to improve women's health? Epidemiol Rev. 2014; 36(1):57-70. [PubMed: 24025350]

24. Bodnar LM, Catov JM, Zmuda JM, et al. Maternal serum 25-hydroxyvitamin D concentrations are associated with small-for-gestational age births in white women. J Nutr. 2010; 140(5):999-1006. [PubMed: 20200114]

25. Fall T, Hagg S, Magi R, et al. The role of adiposity in cardiometabolic traits: a Mendelian randomization analysis. PLoS Med. 2013; 10(6):e1001474. [PubMed: 23824655]

26. Wurtz P, Wang Q, Kangas AJ, et al. Metabolic signatures of adiposity in young adults: mendelian randomization analysis and effects of weight change. PLoS Med. 2014; 11(12):e1001765. [PubMed: 25490400]

27. Shin SY, Fauman EB, Petersen AK, et al. An atlas of genetic influences on human blood metabolites. Nat Genet. 2014; 46(6):543-550. [PubMed: 24816252]

28. Kettunen J, Tukiainen T, Sarin AP, et al. Genome-wide association study identifies multiple loci influencing human serum metabolite levels. Nat Genet. 2012; 44(3):269-276. [PubMed: 22286219]

29. Burgess S, Thompson SG, Collaboration CCG. Avoiding bias from weak instruments in Mendelian randomization studies. Int J Epidemiol. 2011; 40(3):755-764. [PubMed: 21414999]

30. Silverwood RJ, Holmes MV, Dale CE, et al. Testing for non-linear causal effects using a binary genotype in a Mendelian randomization study: application to alcohol and cardiovascular traits. Int J Epidemiol. 2014; 43(6):1781-1790. [PubMed: 25192829]

31. Group HSCR. Hyperglycaemia and Adverse Pregnancy Outcome (HAPO) Study: associations with maternal body mass index. BJOG. 2010; 117(5):575-584. [PubMed: 20089115]

32. Catalano PM, McIntyre HD, Cruickshank JK, et al. The hyperglycemia and adverse pregnancy outcome study: associations of GDM and obesity with pregnancy outcomes. Diabetes Care. 2012; 35(4):780-786. [PubMed: 22357187]

33. Fraser A, Macdonald-Wallis C, Tilling K, et al. Cohort Profile: the Avon Longitudinal Study of Parents and Children: ALSPAC mothers cohort. Int J Epidemiol. 2013; 42(1):97-110. [PubMed: 22507742]

34. Schlemm L, Haumann HM, Ziegner M, et al. New evidence for the fetal insulin hypothesis: fetal angiotensinogen M235T polymorphism is associated with birth weight and elevated fetal total glycated hemoglobin at birth. J Hypertens. 2010; 28(4):732-739. [PubMed: 20075747]

35. Power C, Elliott J. Cohort profile: 1958 British birth cohort (National Child Development Study). Int J Epidemiol. 2006; 35(1):34-41. [PubMed: 16155052]

36. Zhao J, Li M, Bradfield JP, et al. Examination of type 2 diabetes loci implicates CDKAL1 as a birth weight gene. Diabetes. 2009; 58(10):2414-2418. [PubMed: 19592620]

37. Bisgaard H. The Copenhagen Prospective Study on Asthma in Childhood (COPSAC): design, rationale, and baseline data from a longitudinal birth cohort study. Ann Allergy Asthma Immunol. 2004; 93(4):381-389. [PubMed: 15521375]

38. Nohr EA, Timpson NJ, Andersen CS, Davey Smith G, Olsen J, Sorensen TI. Severe obesity in young women and reproductive health: the Danish National Birth Cohort. PLoS One. 2009; 4(12):e8444. [PubMed: 20041193]

39. Olsen J, Melbye M, Olsen SF, et al. The Danish National Birth Cohort--its background, structure and aim. Scand J Public Health. 2001; 29(4):300-307. [PubMed: 11775787]

40. Knight B, Shields BM, Hattersley AT. The Exeter Family Study of Childhood Health (EFSOCH): study protocol and methodology. Paediatr Perinat Epidemiol. 2006; 20(2):172-179. [PubMed: 16466435]

41. Lacroix M, Battista MC, Doyon M, et al. Lower adiponectin levels at first trimester of pregnancy are associated with increased insulin resistance and higher risk of developing gestational diabetes mellitus. Diabetes Care. 2013; 36(6):1577-1583. [PubMed: 23300287] 
42. Jaddoe VW, van Duijn CM, Franco OH, et al. The Generation R Study: design and cohort update 2012. Eur J Epidemiol. 2012; 27(9):739-756. [PubMed: 23086283]

43. Magnus P, Irgens LM, Haug K, et al. Cohort profile: the Norwegian Mother and Child Cohort Study (MoBa). Int J Epidemiol. 2006; 35(5):1146-1150. [PubMed: 16926217]

44. Rantakallio P. Groups at risk in low birth weight infants and perinatal mortality. Acta Paediatr Scand. 1969; 193(Suppl 193):191.

45. Boomsma DI, de Geus EJ, Vink JM, et al. Netherlands Twin Register: from twins to twin families. Twin Res Hum Genet. 2006; 9(6):849-857. [PubMed: 17254420]

46. Medland SE, Nyholt DR, Painter JN, et al. Common variants in the trichohyalin gene are associated with straight hair in Europeans. Am J Hum Genet. 2009; 85(5):750-755. [PubMed: 19896111]

47. Moayyeri A, Hammond CJ, Valdes AM, Spector TD. Cohort Profile: TwinsUK and healthy ageing twin study. Int J Epidemiol. 2013; 42(1):76-85. [PubMed: 22253318]

48. Moayyeri A, Hammond CJ, Hart DJ, Spector TD. The UK Adult Twin Registry (TwinsUK Resource). Twin Res Hum Genet. 2013; 16(1):144-149. [PubMed: 23088889]

49. Speliotes EK, Willer CJ, Berndt SI, et al. Association analyses of 249,796 individuals reveal 18 new loci associated with body mass index. Nat Genet. 2010; 42(11):937-948. [PubMed: 20935630]

50. Dupuis J, Langenberg C, Prokopenko I, et al. New genetic loci implicated in fasting glucose homeostasis and their impact on type 2 diabetes risk. Nat. Genet. 2010; 42(2):105-116. [PubMed: 20081858]

51. Morris AP, Voight BF, Teslovich TM, et al. Large-scale association analysis provides insights into the genetic architecture and pathophysiology of type 2 diabetes. Nat Genet. 2012; 44(9):981-990. [PubMed: 22885922]

52. Teslovich TM, Musunuru K, Smith AV, et al. Biological, clinical and population relevance of 95 loci for blood lipids. Nature. 2010; 466(7307):707-713. [PubMed: 20686565]

53. Ehret GB. Genome-wide association studies: contribution of genomics to understanding blood pressure and essential hypertension. Curr Hypertens Rep. 2010; 12(1):17-25. [PubMed: 20425154]

54. Yaghootkar H, Lamina C, Scott RA, et al. Mendelian randomization studies do not support a causal role for reduced circulating adiponectin levels in insulin resistance and type 2 diabetes. Diabetes. 2013; 62(10):3589-3598. [PubMed: 23835345]

55. Freathy RM, Mook-Kanamori DO, Sovio U, et al. Variants in ADCY5 and near CCNL1 are associated with fetal growth and birth weight. Nat. Genet. 2010; 42(5):430-435. [PubMed: 20372150] 


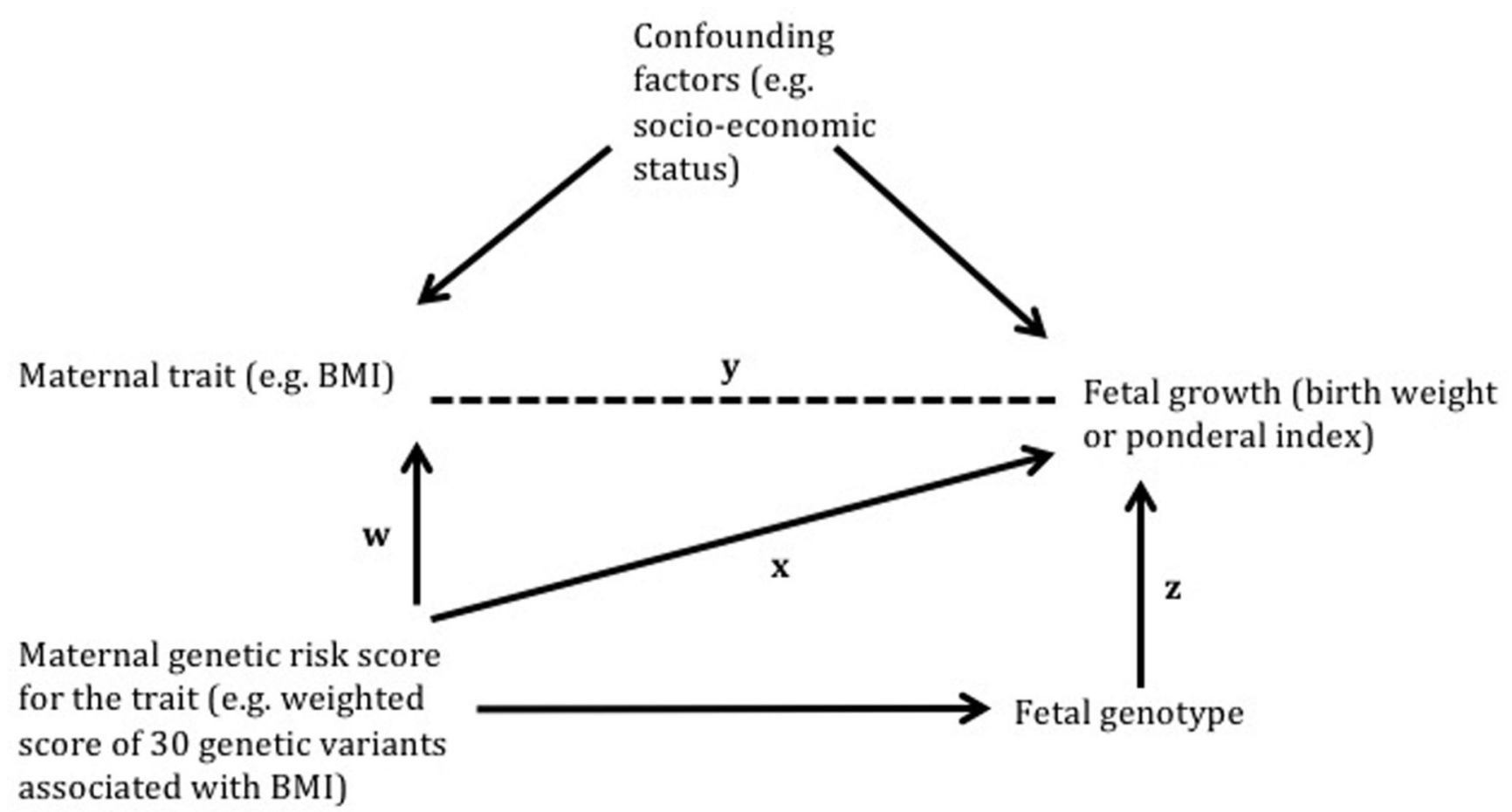

Figure 1.

Principle of Mendelian randomization: If a maternal trait causally influences offspring birth weight, then a risk score of genetic variants associated with that trait will also be associated with birth weight. Since genotype is determined at conception, it should not be associated with factors that normally confound the association between maternal traits and birth weight (e.g. socio-economic status). Estimates of the genetic score-maternal phenotype association (w) and the genetic score-birth weight association (x) may be used to estimate the association between the maternal trait variation that is due to genetic score, and birth weight $(y=x / w)$, which is expected to be free from confounding. If the estimated causal relationship, $y$, is different from the observational association between the measured maternal phenotype and birth weight, this would suggest that the observational association is confounded (assuming that the assumptions of the Mendelian randomization analyses are valid). ${ }^{14}$ The dashed line connecting maternal trait with fetal growth has no arrow, to indicate that the causal nature of the association is uncertain. It is important to adjust for possible direct effects of fetal genotype $(\mathrm{z})$. 


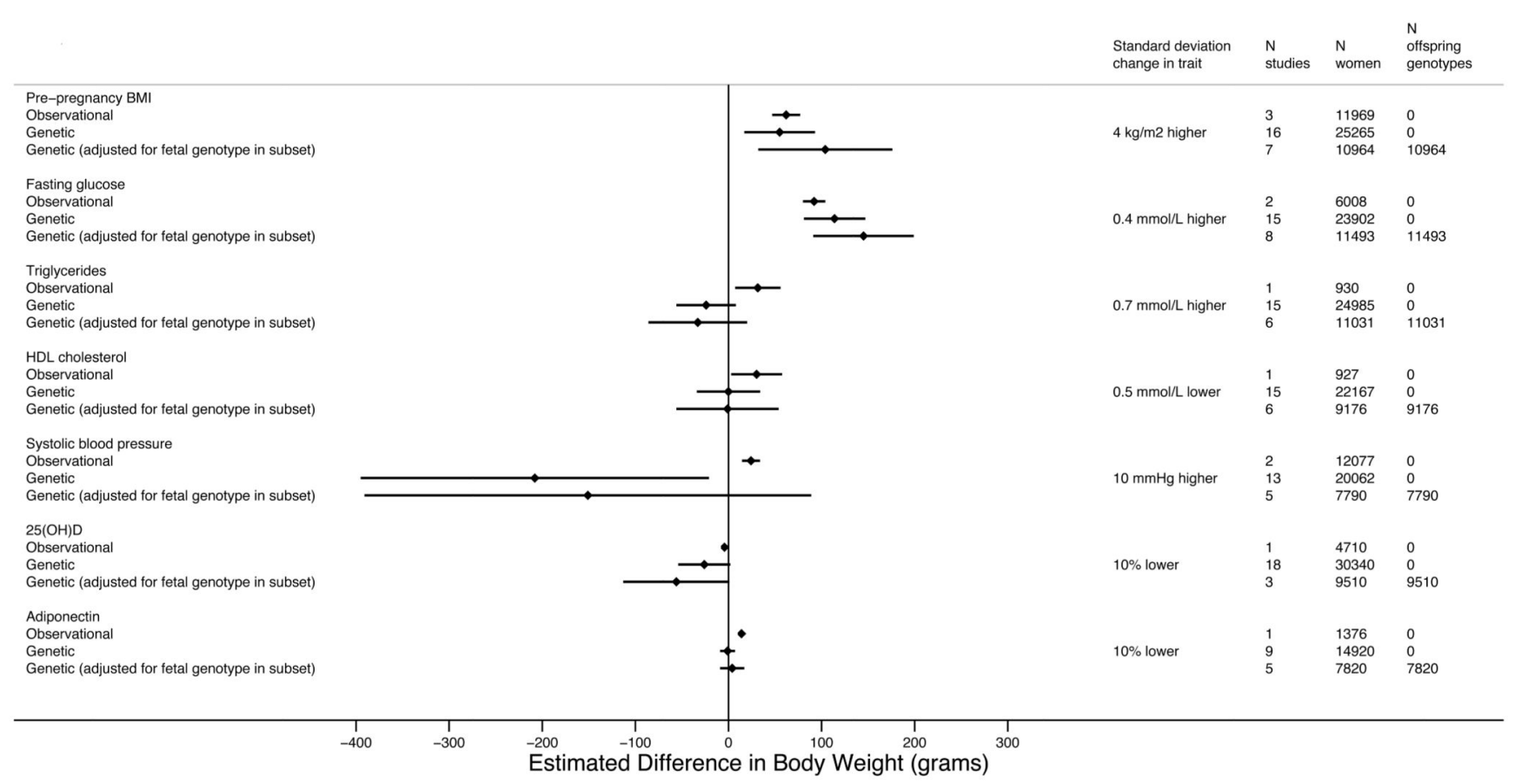

Figure 2.

Comparison of the observational with the genetic change in birth weight (in grams) for a 1 standard deviation (SD) change in each maternal obesity-related trait. For 25[OH]D and adiponectin, we present the change in birth weight for a $10 \%$ change in maternal trait level because these variables were logged for analysis. The genetic change was estimated from Mendelian randomization analysis, in which a genetic score was used to estimate the possible causal relationship between the maternal trait and birth weight. The genetic estimate is presented twice: in the second case it was adjusted for fetal genotype using a subset of available studies. The error bars represent the $95 \%$ confidence intervals around the effect size estimates. For maternal pre-pregnancy BMI and fasting glucose, the $95 \%$ confidence intervals for both the observational and genetic approaches exclude the null, suggesting positive possible causal relationships between maternal BMI and fasting glucose and birth weight. For maternal SBP, the observational analysis suggested a weak positive association with birth weight, whereas the genetic analysis showed evidence of a negative possible causal relationship. Observational analyses suggested that higher maternal triglyceride levels, lower maternal adiponectin and lower maternal HDL-cholesterol levels were associated with higher birth weight, while lower maternal vitamin D status was associated with lower birth weight, but none of these were supported by the genetic analyses. 
Table 1

Key characteristics of participants by study (for full details, see eTable 1)

\begin{tabular}{|c|c|c|c|c|c|c|c|}
\hline Abbreviated study name & Country (sample source) & $\begin{array}{l}\text { Offspring } \\
\text { years of } \\
\text { birth }\end{array}$ & $\begin{array}{c}\mathbf{N} \\
\text { women } \\
\text { with } \\
\text { birth } \\
\text { weight } \\
\text { of one } \\
\text { child }\end{array}$ & $\begin{array}{c}\mathrm{N} \\
\text { offspring } \\
\text { with } \\
\text { genotype }\end{array}$ & $\begin{array}{c}\text { Mean } \\
\text { maternal } \\
\text { age at } \\
\text { delivery in } \\
\text { years (SD) }\end{array}$ & 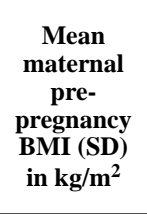 & $\begin{array}{c}\text { Mean } \\
\text { offspring } \\
\text { birth } \\
\text { weight } \\
\text { (SD) in } \\
\text { grams }\end{array}$ \\
\hline ALSPAC mothers ${ }^{33}$ & UK & 1991-1992 & 7304 & 4913 & $28.5(4.8)$ & $22.93(3.73)$ & $3481(475)$ \\
\hline BBC mothers ${ }^{34}$ & Germany & 2000-2004 & 1357 & 1357 & $30.1(5.4)$ & $22.78(3.93)$ & $3472(511)$ \\
\hline B58C-WTCCC ${ }^{35}$ & UK & $1972-2000$ & 855 & NA & $26.2(5.2)$ & NA & $3325(483)$ \\
\hline B58C-T1DGC ${ }^{35}$ & UK & $1972-2000$ & 836 & NA & $26.1(5.4)$ & NA & $3379(469)$ \\
\hline CHOP mothers ${ }^{36}$ & USA & 1987-present & 312 & NA & NA & NA & $3440(562)$ \\
\hline COPSAC-2000 mothers ${ }^{37}$ & Denmark & $1998-2001$ & 282 & 282 & $30.4(4.3)$ & NA & $3560(505)$ \\
\hline DNBC-GOYA $^{38}$ & Denmark & $1996-2002$ & 1805 & NA & $29.2(4.2)$ & $23.57(4.27)$ & $3643(495)$ \\
\hline DNBC-PTB-CONTROL ${ }^{39}$ & Denmark & 1987-2009 & 1649 & 975 & $29.9(4.2)$ & $23.57(4.27)$ & 3595 (497) \\
\hline EFSOCH mothers ${ }^{40}$ & UK & $2000-2004$ & 746 & $332^{*}$ & $30.5(5.3)$ & $24.07(4.42)$ & $3512(480)$ \\
\hline GEN-3G mothers ${ }^{41}$ & Canada & 2010-2013 & 676 & NA & $28.4(4.4)$ & $24.83(5.63)$ & $3448(433)$ \\
\hline Generation R mothers ${ }^{42}$ & The Netherlands & $2002-2006$ & 3810 & 2196 & $31.2(4.5) \dagger$ & $23.12(3.92)$ & $3528(494)$ \\
\hline HAPO mothers (GWAS $)^{5}$ & UK, Canada, Australia & 2000-2006 & 1380 & 1300 & $31.5(5.3) \dagger$ & $24.5(5.0)$ & $3557(517)$ \\
\hline HAPO mothers (non-GWAS) ${ }^{5}$ & $\begin{array}{l}\text { USA, UK, Canada, } \\
\text { Australia }\end{array}$ & $2000-2006$ & 3590 & 2318 & $30.4(5.4)^{\dagger}$ & $24.63(5.33)$ & $3526(463)$ \\
\hline MoBa mothers ${ }^{43}$ & Norway & 1999-2008 & 650 & 350 & $28.5(3.3)$ & $23.93(3.94)$ & $3679(430)$ \\
\hline NFBC1966 44 & Finland & $1987-2001$ & 2035 & NA & $26.5(3.7)$ & NA & $3525(461)$ \\
\hline $\mathrm{NTR}^{45}$ & The Netherlands & $1946-2003$ & 706 & NA & $27.1(3.7)$ & NA & $3469(529)$ \\
\hline $\mathrm{QIMR}^{46}$ & Australia & $1929-1990$ & 892 & NA & $24.5(4.0)$ & $22.79(5.13)$ & $3344(532)$ \\
\hline TwinsUK $^{47,48}$ & UK & NA & 1602 & NA & NA & NA & $3365(581)$ \\
\hline
\end{tabular}

NA, not available.

* Expanded study names: ALSPAC, Avon Longitudinal Study of Parents and Children; BBC, Berlin Birth Cohort; B58C-WTCCC, 1958 British Birth Cohort-Wellcome Trust Case Control Consortium; B58C-T1DGC, 1958 British Birth Cohort-Type 1 Diabetes Genetics Consortium; CHOP, Children's Hospital Of Philadelphia; DNBC-GOYA, Danish National Birth Cohort-Genetics of Obesity in Young Adults study; DNBC-PTBCONTROLS, Danish National Birth Cohort Preterm Birth study Controls; EFSOCH, Exeter Family Study Of Childhood Health; GEN-3G, Genetics of Glycemic regulation in Gestation and Growth; HAPO, Hyperglycemia and Adverse Pregnancy Outcome study (GWAS, Genome-Wide Association Study); MoBa, the Norwegian Mother and Baby Cohort; NFBC1966, the Northern Finland 1966 Birth Cohort; NTR, Netherlands Twin Registry; QIMR, Queensland Institute of Medical Research.

In Generation R, maternal age was recorded, on average, at 14.4 weeks of gestation; in HAPO, maternal age was recorded, on average, at 28 weeks of gestation.

${ }^{*}$ Fetal genotype in EFSOCH available only for the fasting glucose genetic score. 


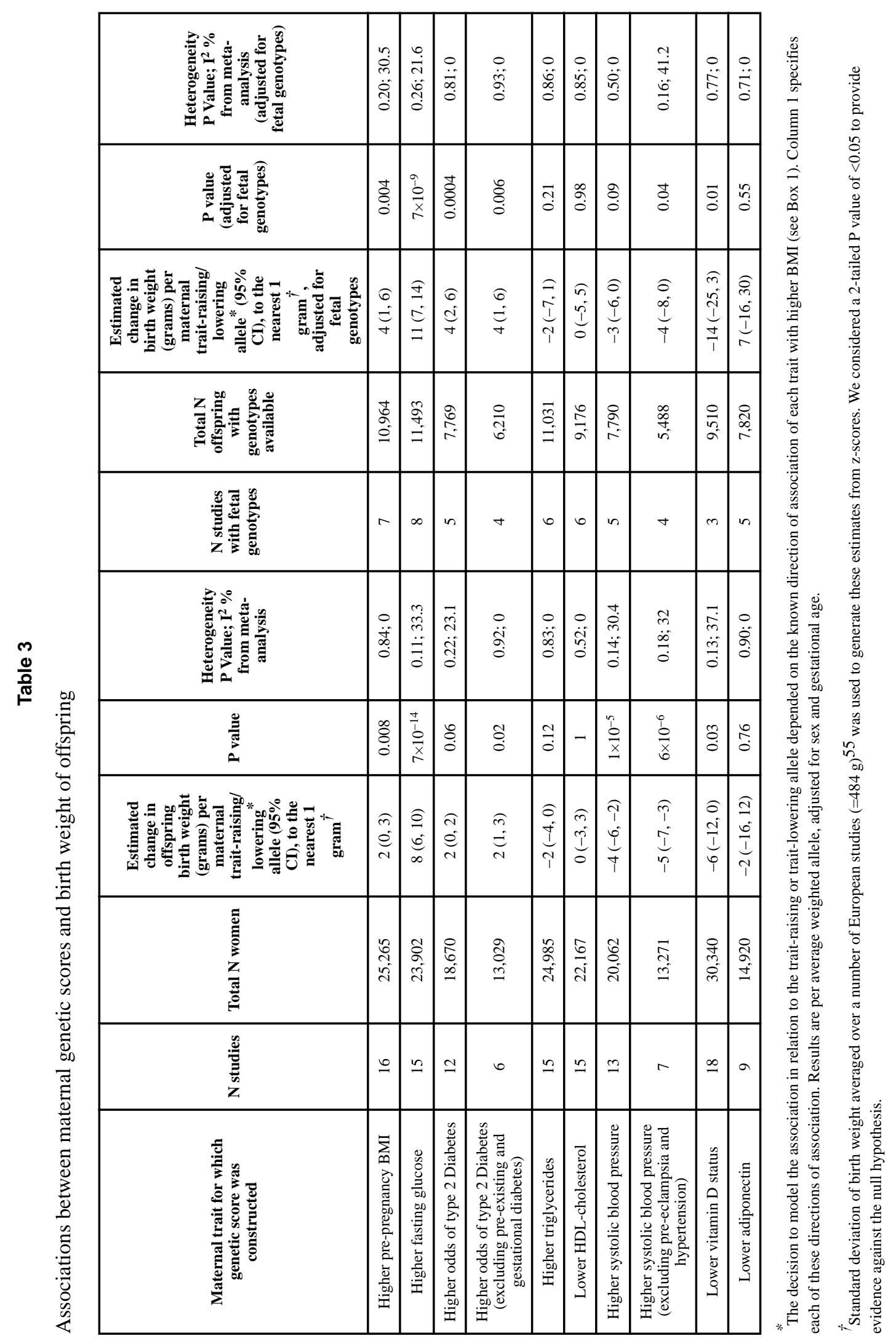


